\title{
Design and Implementation of an Emergency Department (ED) Based Rapid HIV Screening Program
}

\author{
Fredric Hustey*, Michael Phelan, Sharon O'Keefe and Tracy Barbour \\ Cleveland clinic, Cleveland, $\mathrm{OH}$, USA
}

\section{Objective}

To design and implement an ED based rapid HIV screening program targeting high risk patients presenting with signs, symptoms, or concerns for sexually transmitted diseases; to determine the prevalence rate of HIV infection in the tested population; to determine the proportion of HIV-positive patients with successful linkage to outpatient care after ED discharge.

\section{Introduction}

In 2003, the Centers for Disease Control and Prevention (CDC) in Atlanta, Georgia estimated that approximately 1 million people in the United States were living with HIV/AIDS, and that approximately $25 \%$ of these were undiagnosed and unaware of their HIV infection. For many such patients the ED may be the only part of the health care system that is utilized. In 2006, the CDC revised their recommendations for HIV testing in a variety of care settings including the ED. In spite of this change, most EDs throughout the United States still do not offer routine HIV testing. Implementing successful ED based testing models may lead to greater acceptance of ED based testing, earlier detection, and further reduction in the transmission of HIV in the United States $(1,2,3,4,5)$.

\section{Methods}

This was a prospective cohort study targeting ED patients presenting with signs, symptoms, or concerns for sexually transmitted diseases. The emergency department is part of a large urban tertiary care center with approximately 55,000 ED patient visits per year. In November of 2011, an ED based POC HIV testing program was initiated using the Clearview HIV Stat-Pack (blood sample) targeted towards patients presenting with signs, symptoms, or concerns for sexually transmitted diseases. Patients were recruited and consented for testing by ED clinical staff. All records regarding testing were recorded in the ED point of care lab at the time of the test (per standard lab operations) and then subsequently entered into a secure electronic database by trained research personnel. This database was used to determine the prevalence of HIV positivity in the tested population. For patients testing positive in the emergency department, liaison social worker followed up via patient telephone interview at one week and one month intervals (if there was no linkage established at the initial one week interview) to establish whether there was linkage to care after ED discharge. Successful linkage to care was defined as followup in infectious disease clinic within one month. Proportions with $95 \%$ confidence intervals (CI) are reported.

\section{Results}

Between November 29th, 2011 and August 18th, 2014 a total of 1090 patients underwent ED based POC HIV testing. 43\% (467/1090) were male, 74\%(804/1090) African American, and 0.4\% (4/1090) Hispanic. Mean age was 27 years. 32 patients tested positive of which 31 were confirmed to be HIV positive by confirmatory testing (3\%; $95 \%$ CI, 2-4\%). 1/32 was considered to be false positive based on follow up Western Blot and viral load testing. 27/31 patients were previously undiagnosed (87\%; 95\%CI, 71-95\%), while 4 patients had reported prior positive results performed at other sites.100\%
(32/32: 95\%CI, 87-100\%) of POC HIV positive patients were linked to outpatient care.

\section{Conclusions}

A targeted POC HIV testing program is feasible in the emergency department setting. Targeting high risk populations such as those presenting with signs, symptoms, or concerns for sexually transmitted diseases yielded much higher HIV prevalence rates than previously reported among ED populations. HIV positive patients can routinely be linked to initial outpatient care from the ED setting with acceptable rates of follow up.

\section{Keywords}

HIV; detection; prevalence

\section{Acknowledgments}

This project was supported in part by grant from the Ohio Department of Health and Centers for Disease Control.

\section{References}

1. Glynn M, Rhodes P. Estimated HIV prevalence in the United States at the end of 2003. National HIV Prevention Conference; June 2005;

Atlanta. Abstract T1-B1101. Available at: http://www.aegis.com/ conferences/

NHIVPC/2005/T1-B1101.html. Accessed February 11, 2008.

2. CDC MMWR, August 8, 2008 / 57(31);845-849 http://www.cdc.gov/ $\mathrm{mmwr} / \mathrm{preview} / \mathrm{mmwrhtml} / \mathrm{mm} 5731 \mathrm{a} 1 . \mathrm{htm}$, accessed June 25, 2013

3. Branson BM. Revised recommendations for HIV testing in healthca re s ettings. Available at: http://www.cdc.gov/hiv/topics/testing/ resources/slidesets/pdf/

4. USPSTF Recommendations for STI Screening, http://www. uspreventiveservicestaskforce.

org/uspstf08/methods/stinfections.htm

5. CDC 2010 STD Treatment Guidelines, http://www.cdc.gov/std/ treatment/

201

*Fredric Hustey

E-mail: husteyf@ccf.org 